\title{
Bumps in the Road
}

\section{James B. Breckinridge, Breckinridge Associates, LLC, College of Optical Sciences, The Univ. of Arizona, California Institute of Technology}

\begin{abstract}
I have two subjects I want to talk about today - one is on some historical experiences, and the other is about mistakes made in the development of high-performance optical systems, develop of functional requirements and flow-downs, identification of design approaches for an instrument, etc. One thing I'm working on relates to polarization and how it affects radiometry and the image quality of an optical system and so we'll spend a little bit of time talking about that. Finally, though the HST failure has been widely covered, a few additional comments are probably also worthy of mention.

Let's begin with some of the work I did in collaboration with Keith Patterson, when he was a graduate student at Caltech. Polarization is important to science as it measures asymmetry in the universe. Polarization also affects image quality in everyday cameras, especially when you get down to the diffraction limit. As an example, see Figure 2, which shows a point spread function of a young star (HR4796A) from a recent ( $<18$ month old) first-light photo taken by the Gemini planet imager. The light scattered by the dust disk orbiting the star is evident, but with a linear polarizer as used in the right-side image, much of the dust cloud disappears. If you look very carefully, you can see the angle of inclination of the orbital plane of the dust in the left image, and angle of inclination of the polarized material in the right image, are not equal. This is a current big mystery in astrophysical circles.
\end{abstract}

Anyway, moving on to the polarization aberrations in modern space telescopes, for incident unpolarized white light, light at the focal plane is always partially polarized. You can't get around that because of the way we package our optical systems, particularly large ground-based telescopes that are $\sim F / 1.2$ to F/1. The giant Magellan Telescope primary is going to be $\sim F / 0.7$. Shortly, you'll see why F-numbers are important here.

In the case that we looked at, the point spread function bifurcates to two point spread functions, one for each polarization. These are about 10-mili-arcseconds apart, so if you're making milli-arcsecond type measurements, polarization is very important .These effects are not isoplanatic, and the symmetry of the point spread function changes across the field of view. The polarization wavefront map shows about a fortieth of a wave of wavefront error and this cannot be easily corrected by adaptive optics (without an operational cost) because the adaptive optics are insensitive to polarization. If you correct this with adaptive optics by inserting a beamspliiter and a polarizer and separating your beam into two orthogonal polarization states, you wind up wasting precious light. Of course we'd all like to invent a way to correct our beams but not waste light. The point of this is that these may all be very small effects, but they still can decrease your signal-tonoise ratio.

An Optical Believe It or Not: Key Lessons Learned III, edited by Mark A. Kahan, Proc. of SPIE Vol. 9197, 919706 · (C) 2014 SPIE · CCC code: 0277-786X/14/\$18 · doi: 10.1117/12.2066922 
So what's the source of the polarization? Figure 6 gives a simple diagram of an optical system with something to the left in object space, and then light reflecting off of three mirrors to an image plane. We pretty-much always have reflections in order to fit the available package size/volume, but each one of those reflections introduces polarization. Here we've shown things all in one plane so different input angles all have similar polarization effects, but when there are compound angles in the system, a large amount of circular polarization can be introduced, which behaves differently than a linear polarization. So what's the source of this polarization? Well if we take bare aluminum (see Figure 7), and the enemy is wavelength and the angle of incidence. $R_{s}$ and $R_{p}$ can differ very greatly. (There are analogies here to seeing through dielectric windows in a building and looking through a car window with polaraized sunglasses.) Metals and metallic coatings can be even more insidious because they introduce an imaginary part of the index of refraction which results in wavefront phase retardation before you get some circularly polarized light. You see this in the right hand side of Figure 7 which plots this retardation in radians as a function of incident angle from zero to eighty degrees. So you really have to remember that all optical systems manipulate the complex amplitude of the wavefront all the way to the focal plane. You don't actually have an intensity until you produce the modulus square of all those phases and amplitudes propagating to the focal plane and that happens when the complex wavefront is turned into energy or power at the focal plane by the physical detection process.

Here's a little exercise from a class I teach. I have my students take an F/8 primary mirror, followed by a fold-mirror, and calculate the polarization aberrations at four points in the field of view ( $A, B, C, \& D$ in Figure 8 ), where point-B is on-axis. At $F / 8$ the the fold mirror adds different results at different positions in the FOV. If you stand back at the focal plane and look back at the pupil, the pupil appears polarization apodized.

In Figure 9 we have an exit pupil on the left, and on the right we have the image-plane \& PSF. Things look nice and symmetrical in the top two images. Now if I take two sheets of linear polarizing material and cover $1 / 2$ the aperture with a piece of the polarizing sheet, but arrange the two sheets to be orthogonal to each other, I get an incoherent superposition of two PSFs, and we have a resultant PSF/resolution that now has an angular dependence. So if I have anything in my optical system that introduces asymmetry and that introduces polarization into the optical system, l'll wind up taking a resolution hit. Figure 10 shows the math of all this and the impacts of polarization in terms of wavefront shear or retardance and diattenuation. Even if we could somehow build/package a system containing all normal incidence mirrors, just the anisotropy in deposited thin films can change the polarization reflectivity across the surface of a mirror. The left most image in Figure 11 shows lengths of lines whose radial extent is proportional to the deviation of the reflected ray. In the middle part of the Figure, line lengths are proportional to diattenuations which show the larger phase changes that occur near the edges of the pupil. The right Figure shows the sagittal retardances.

So, again, what are the sources of internal polarization that modify image quality? Well, there's the Fresnel effects (see the equations we showed in Figure 10, which were derived 
by Fresnel about 40-years prior to the work of Maxwell), and there's the effects that come about from highly reflecting metal thin-film anisotropies. Some of these later effects come about from the film deposition process. Astronomers almost always need to deposit mirror coatings in chamber configurations called clam-shells because of the lack of volume. A few years ago, one of Angus Macleod's students deposited aluminum at various angles, and he then measured the polarization content of normally incident reflected light. $\mathrm{He}$ discovered that even if you input unpolarized normally incident light, the reflected light comes back polarized, where the polarization depends upon the angle at which you made the deposition! So even if you evaporate something in a small clamshell. Your evaporators are seeing at a steep angle, and possibly some polarization will be introduced. And of course we also have to account for the effects of lenses, dielectric mirrors, beamsplitters, and birefringence and particularly the effects of tilted components.

In Figure 13 we look at a "typical" astronomical telescope. Its F/1.2 primary mirror has a clear aperture of $2.4 \mathrm{M}$, and it sits in a system with a FOV of $1^{\circ}$. Figure 14 shows the vector phasemap. There's about a $5^{\circ}$ phase change over the pupil which is about a sixtieth of a wave is for just one fold mirror. For two fold mirrors the error will be about a thirtieth of a wave and it's not the same for both polarizations. "Figure" 15 gives a table which summarizes the calculations, and you can see how the percentage polarization changes the point spread function. The point spread functions for different polarizations are displaced from each other, and together they appear to produce an elliptical/asymmetric zone.

One interesting point here is that each 3-D opto-mechanical layout will have different polarization properties depending on the coatings, so you can't say which, and by how much, a specific high-acuity system is really best until a physical optics analysis is completed.

So that's the polarization Lesson Learned. A telescope is a polarization filter of sorts, and it's not so simple to get the radiometry precisely right, and calibrations need to account for where you are in the FOV.

Another interesting polarization point that ties to nearly all large ground-based telescopes which are alt-az mounted is polarization effects will depend on where the telescope is pointing in the sky. This is often ignored or neglected, and may not be well understood by those less familiar with polarization effects. The question is, does it make a difference to your science; does it make a difference to how well you're tracking a missile? You need to run through the signal-to-noise ratio calculations and assess things. A Lesson Learned should be "If in doubt, calculate it out!"

OK, now let's move from polarization to another subject I want to talk briefly about, and that's a few of the Lessons Learned from the Hubble Space Telescope (circa 1990). A overly simplified Lesson would be to "measure twice, cut once." We need to keep remembering these lessons learned from the Hubble so we don't repeat past errors in the future. A mantra I used when I was managing a section of optical engineers was that if you designed it, you're responsible for making it work. You want active feedback to the designer so he or she can participate enough to take ownership in the success of the project. This is extremely important. 
Many optical manufacturing companies will job-shop out the design, and when they get around to doing the final testing, maybe five years later, the optical designers are gone and the new crew may not understand what subtle things to look for, or what seems anomalous.

Clearly identify your single point failures. If it's really important. use all the tools in your toolkit, ray-trace design, scalar diffraction, diffraction vector diffraction, polarization analysis, etc. Don't let threats of cancellation rattle you. The Hubble Space Telescope project hit this sort of monthly, or at least every six months. Managing a program through negative reinforcement isn't conducive to bringing out the best work folks can do. Encourage employees to do well. Negative reinforcement can totally demoralize everybody and prevent open discussions of inconsistent results (on HST there were multiple test results that were effectively "ignored" that showed there was a problem). You then get accidents and hardware surprises. When we reviewed the HST's original ("Fossil") data, It didn't take terribly long to unearth the problem once things were discussed and cross-compared. Sometimes even a peer-review team will get lost in minutia, while the elephant in the room remains unnoticed. You have to be very careful and have a good sixth sense to know what to listen for, and to be sure you're teasing out the Devil in the Details, but not ignoring big-picture issues.

NASA formed the official HST Failure Review Board on 2 July 1990 and the presentation to Congress and the report was published on 19 November 1990, less than five months after commissioning the Failure Board. Prescription Retrieval started in August of 1990. Sadly the many boxes of notebooks that covered the HST had been taken to the dump two months before launch, because there was a dispute between NASA and the contractor over who was going to pay for storage of the boxes! Fortunately some of the hardware and test equipment used in the build was still (after 9-years) in bonded stores at the contractor, and individual engineers had kept a lot of important data in their personal notebooks, and we were able to use that information very nicely. The science community was irate. The pressure to quickly but carefully determine what went wrong \& how to institute a fix was high.

Figure 24 shows the primary mirror build process, and the use of the refractive and reflective null correctors, and the testing done to establish the mirror's center of curvature. (l'll skip through a number of these charts as this is a well presented subject, but I wanted to include them here for completeness.) [The differences between the testing done with the refractive null corrector and the reflective null corrector showed the spherical aberration, as did several other tests. Though the reflective null was a better test on-paper, the reflective null corrector was fabricated incorrectly when one of the mirrors in the null corrector was located incorrectly (a metering rod's end-cap was used to set a length instead of the road itself).] Test results were purposely isolated from each other, and only specific aspects of individual tests were reviewed. Sadly though this speeded the review of test results, it also led to this resulted in missing one the elephants in the room (curved fringes at the edge of an interferogram which showed spherical aberration).

There was also an interesting management error. We discovered that the designer was not invited to climb the test tower to verify that his test worked the way he had expected it should. 
There was a firewall between the designers/mathematicians and those who were building the hardware, and management (both at the contractor and at NASA) didn't have the technical skills and/or involvement needed to bridge the gap. There were several places where the error could have been caught, be it differences seen between the Refracting, Reflecting and Inverse Null Correctors, Metering Road/Spacer anomalies. There were other clues as shown in my charts, but these were also ignored.

Anyway, HST is now going strong using it's corrective lenses, and 30-years since construction, 22-years on-orbit, and 4-Re-Servicing Missions later, it's still rewriting science books and leading to new discoveries. In this regard, there's another Lesson here. When faced with adversity, examine the situation, and, if it's important enough, don't give up!

That's my talk. Thank you for listening!

[Please Note: This paper has an SPIE approved but somewhat modified format as it is a transcript from a video we made of Jim Breckinridge's excellent talk. It has been edited here and there by the Session Chair to better fit this archival/written format, and the Chair apologizes to both the author and the reader for any inadvertent transcription errors. The interested reader is encouraged to view the full video/audio recording of this talk, which also includes all the Figures which become synced to the text just by watching the presentation. The chair did not insert Figure numbers into this text as the process became too labor intensive. Again, the video/audio is planned to be available as an SPIE download. MK-] 


\section{Mistakes in instrument development}

- Create high performance optical system

\section{Bumps}

Monday 18 August 2014

SPIE: $9197-4$

\author{
James B Breckinridge \\ Caltech
}

1. functional requirements

2. system requirements

3. design approach Polarization: radiometry \& IQ

4. design

5. Specifications

6. Hardware build

7. Integration \& Tes

8. calibration - verification of the system design

\section{Polarization}

- In collaboration with Keith Patterson, graduate student CALTECH

- Polarization is important to science

- Polarization affects image quality

- Radiometry \& PSF shape

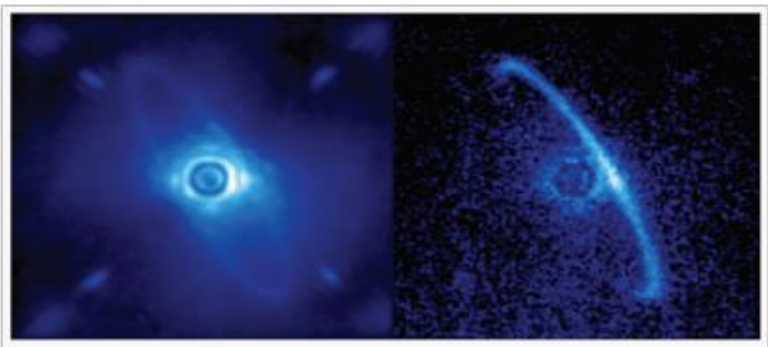

Figure 2. Gemini Planet Imager's first light image of the light scattered by a disk of dust orbiting the young star HR4796A. This narrow ring is thought to be dust from asteroids or comets left behind by planet formation; some scientists have the regid residual light from the central star scattered by turbulence in the Earth's the bagh is of the disk is strongly polarized as it scatters towards us.

Image credit: Processing by Marshall Perrin, Space Telescope Science Institute. s/tEfHIll Resolution JPG

\section{Polarization aberrations in modern space telescopes}

\section{What is the source of polarization?}

- For white-light unpolarized radiation incident on the telescope

- Light at the focal plane is partially polarized

- PSF bifurcates: 0.010 marcsec

- System is not isoplanatic,

- PSF shows an ellipticity of 0.05

- Polarization wavefront map shows $\sim 1 / 40$ wave error - not correctable by $\mathrm{A} / \mathrm{O}$

- These may be small effects - do they decrease science SNR?

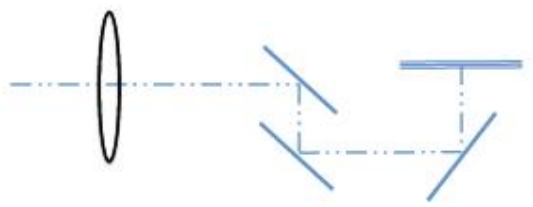




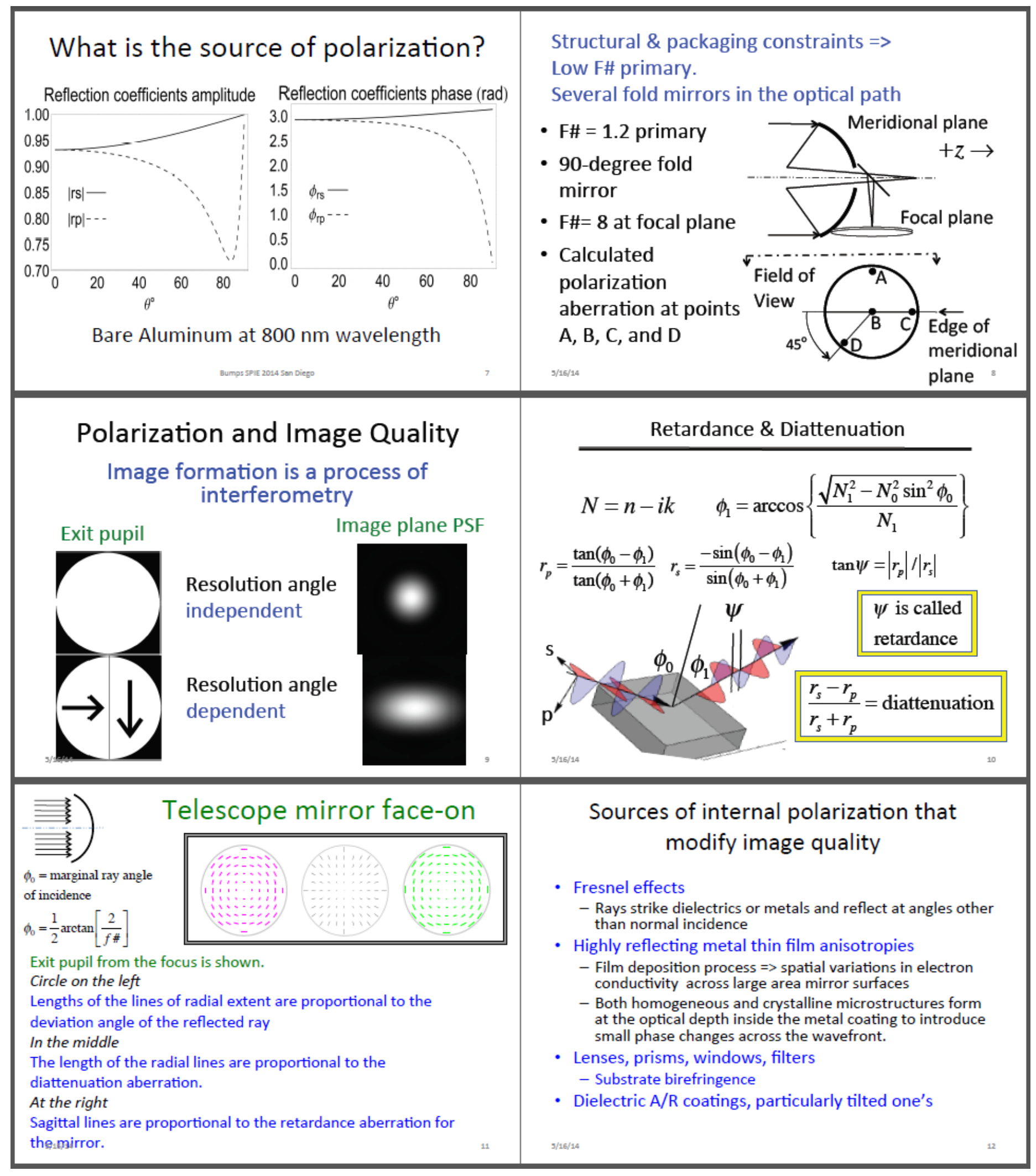




\section{Typical astronomical telescope}

- Table of parameters

\begin{tabular}{|l|l|}
\hline & \\
\hline Primary clear aperture & 2.40 meter \\
\hline Primary F\# & 1.20 \\
\hline Cassegrain focus F\# & 8.0 \\
\hline Field of View (diameter) & 1.0 degree \\
\hline High reflective metal coating & Bare fresh aluminum \\
\hline Wavelength & $800 \mathrm{~nm}$ \\
\hline
\end{tabular}

\section{PSF summary table $290^{\circ}$ reflections}

\begin{tabular}{|c|c|c|c|c|c|c|}
\hline \multirow{5}{*}{ On axis } & $\begin{array}{l}\text { s Polarized } \\
\text { PSF Peak }\end{array}$ & $\begin{array}{c}p \\
\text { Polarized } \\
\text { PSF Peak }\end{array}$ & $\begin{array}{c}\text { PSF peak } \\
\text { shear in } \\
\text { arcseconds }\end{array}$ & $\begin{array}{l}\text { PSF shift } \\
\text { in units } \\
\text { of PSF } \\
\text { half } \\
\text { width } \\
\end{array}$ & \begin{tabular}{|l|} 
Angle \\
between \\
the two \\
PSF's in \\
degrees
\end{tabular} & $\begin{array}{l}\text { Ellipticity } \\
\text { of } \\
\text { I\{PSF(s) - } \\
\text { PSF(p)\} } \\
@ \text { fwhm }\end{array}$ \\
\hline & 12.2 & 10.0 & .010 & & 7.13 & .0530 \\
\hline & 12.0 & 9.9 & 0.09 & & 3.58 & .0525 \\
\hline & 12.0 & 10.0 & 0.08 & & 3.58 & .0522 \\
\hline & 12.0 & 9.6 & .010 & & 0.00 & .0524 \\
\hline
\end{tabular}

Note

- That these numbers are for a Cassegrain focus camera

with two $90^{\circ}$ reflections in front of the focal plane

- The science instrument often adds 2 more 90-degree reflections and a low F\# relay optic, which may double the entries

- Each 3-D opto-mechanical layout will have different polarization properties

By the time we include the fold mirrors and relay optics in the instrument this error could be up to $1 / 10$ wave that cannot be corrected using existing technology

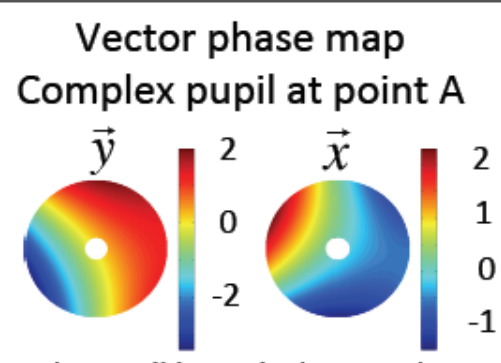

$5 \operatorname{deg} \Delta \phi$ over the pupil is equivalent to about $1 / 60$ wave error for 1 fold mirror; 2 mirrors $=>1 / 30$ wave Not the same for both polarizations and cannot be fixed with $\mathrm{A} / \mathrm{O}$.

Spatially variable retardance plate (SVRP) will fix the Fresnel aberrations.

\section{Summary}

- Even if the source is not polarized, the telescope and instruments (including a coronagraph) are "polarization filters" $=>$ wrong radiometry \& poor image quality unless you pay attention to this fact

- PSF's become asymmetric

- Polarization transmissivity of large ground based telescopes depends on where in the sky the telescope is pointing.

- But does it make any difference - the requirements on the SNR drives the polarization requirement

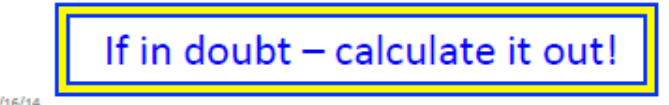

\section{Here we will discuss:}

- The optical fabrication and test processes

- Requirement \& error

- Fossil hardware, notes and interferograms

- The magnitude and sign of the error in the on-orbit telescope

- The WF/PC Fix

- Identify the four tests that suggested an error before launch 


\section{More facts}

- NASA formed the official failure review board July 2

- Presentation to congress \& report published Nov 1990 (5 months from start)

- Prescription retrieval started August 1990

- By the spring of 1991, 7 teams using independent methods gathered to agree on the on-orbit telescope prescription so the optical correctors could be made
Level 1 Optical system specification: Measured (on orbit) encircled energy

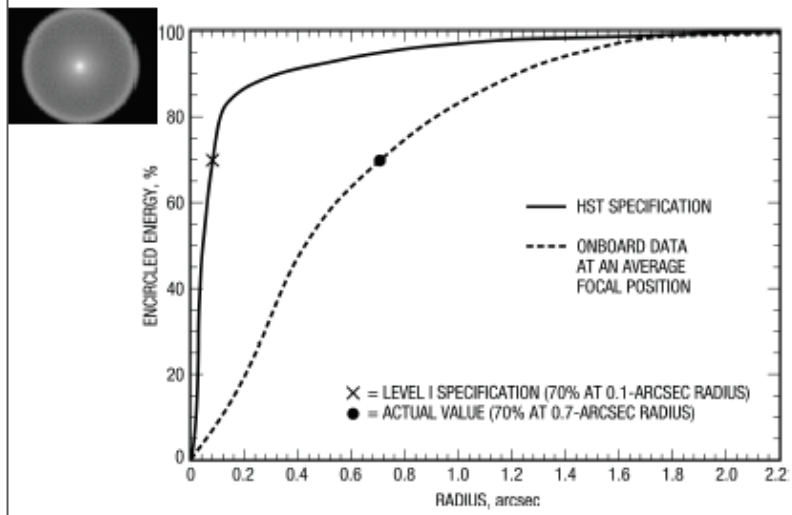

\section{Failure Board Charter}

- Working group to review, analyze and evaluate facts and circumstances regarding the manufacture,

development and testing of the Optical Telescope Assembly

- Determine how \& when the problems in the OTA occurred

- Determine how this aberration could go undetected prior to launch

- Not established to render, advise or make recommendation

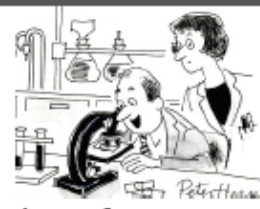

\section{.}

(1) . 


\section{Primary Mirror Processing (1)}

- One facility accepted the blank and

- Rough \& fine-ground the concave front surface to near net shape

- Designed and manufactured a two lens refractive null corrector which used spherical and planar surfaces - very simple \& less room for error

- Tested the fine ground surface using the refractive null \& shipped the mirror to another facility for polishing \& figuring

\section{Primary Mirror Processing (2)}

- The second facility accepted the rough ground mirror

- Designed and built a special purpose reflective null corrector for testing to the UV

- Completed figuring and final polish in April 1981 using the reflective null $\lambda / 100$

- Radius of curvature was verified with the refractive null.

- Interferogram shows the spherical aberration error, but not recognized! That was not the purpose of the test!

- Peer-review panel did not review this material

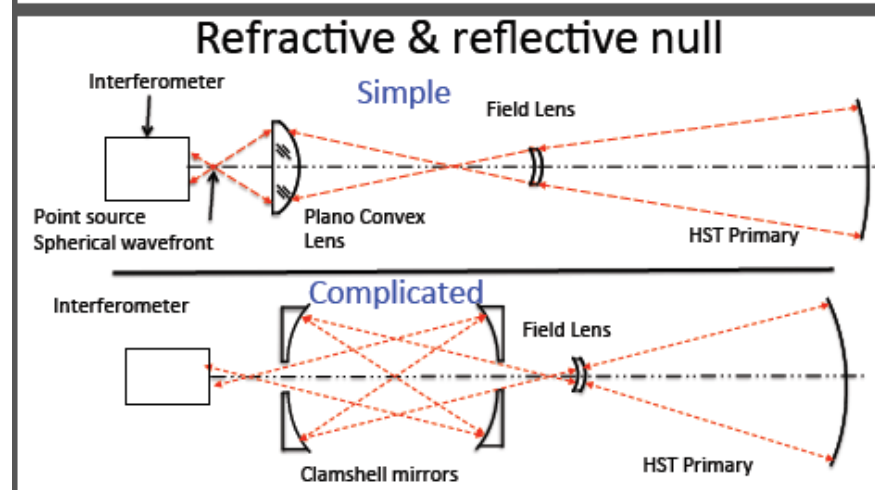

The refractive nulls use transparent glass.

Predicted they could not meet the specification because

Of non-uniform index of refraction in the glass.

Therefore the complicated reflective null was required ${ }_{7}$

\section{The reflective null The Inverse Null}

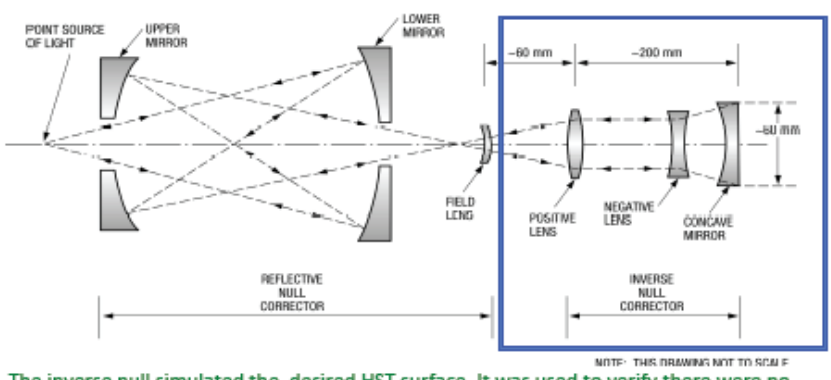

rse null simulated the desired HST surface. It was used to verify there were n drifts in the Reflective null during figuring. The error should have been visible here, but the optician was told only to look for changes. The clamshell was the "absolute reference ${ }^{n}$ - not the inverse null!
Fault tree analysis of the reflective null corrector indicated possible error sources

- Field lens inserted backward

- Wrong index of refraction glass used in the field corrector

- Optical elements incorrectly spaced

- CAD analysis quickly showed only an error in spacing of the all-reflecting null was the most likely source.

\section{Primary mirror as delivered from rough grind to figuring}

- Refractive null corrector

- Straight-line fringes show that the primary was figured correctly in the rough grinding phase.

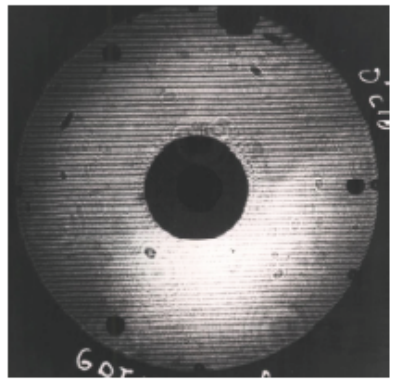




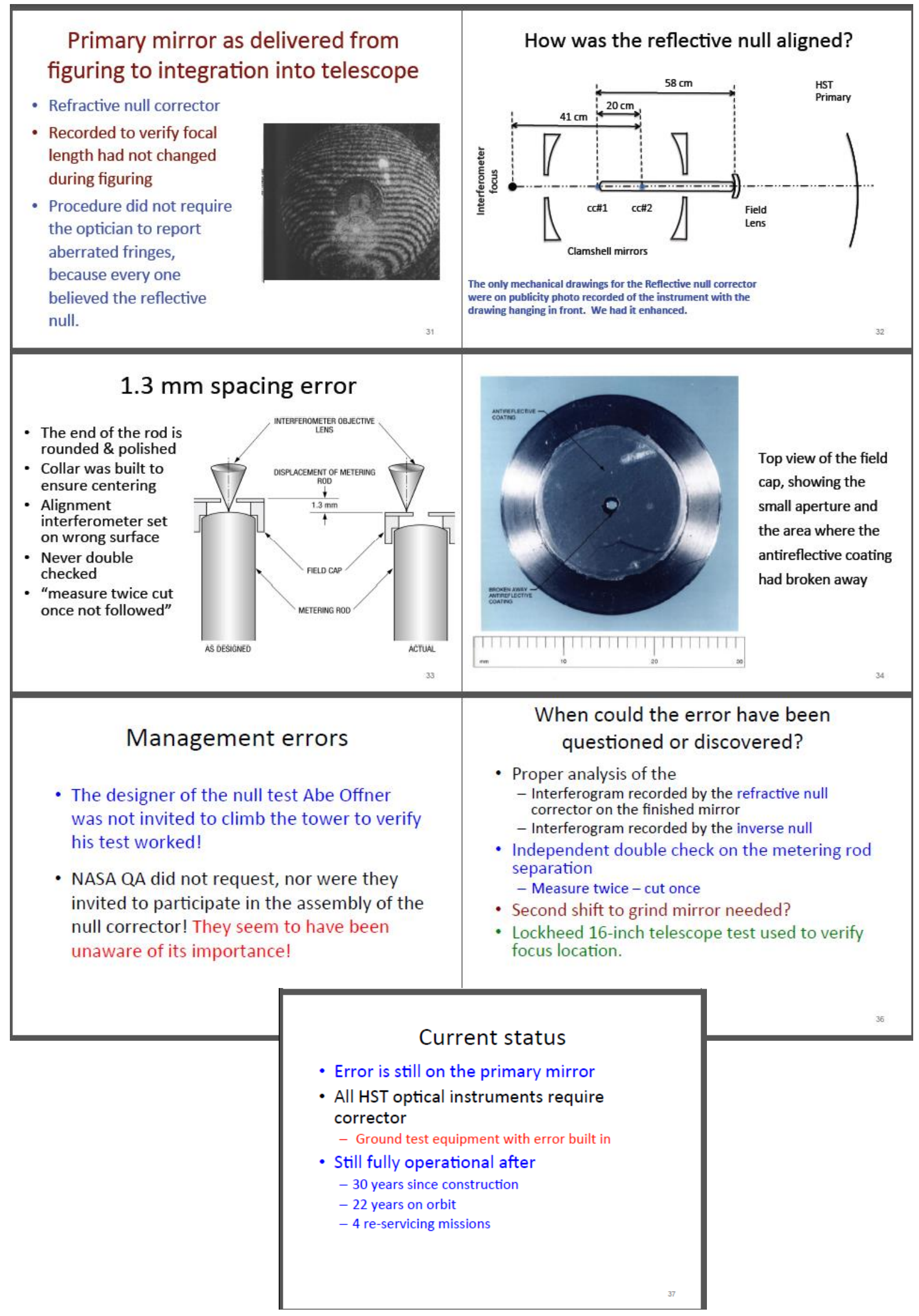

